\title{
Asymptomatic Construct Failure after Metastatic Spine Tumor Surgery: A New Entity or a Continuum with Symptomatic Failure?
}

\author{
Naresh Kumar, Ravish Patel, Barry Wei Loong Tan, Jiong Hao Tan, Naveen Pandita, Dhiraj Sonawane, \\ Keith Gerard Lopez, Khin Lay Wai, Hwee Weng Dennis Hey, Aravind Kumar, Gabriel Liu \\ Department of Orthopaedic Surgery, National University Health System, Singapore
}

Study Design: Retrospective cohort study.

Purpose: To study the incidence, onset, underlying mechanism, clinical course, and factors leading to asymptomatic construct failure (AsCF) after metastatic spinal tumor surgery (MSTS).

Overview of Literature: The reported incidence rates for implant and/or construct failure after MSTS are low (1.9\%-16\%) and based on clinical presentations and revisions required for symptomatic failures (SFs). AsCF after MSTS has not been reported.

Methods: We conducted a retrospective analysis of 288 patients (246 for final analysis) who underwent MSTS between 2005-2015. Data collected were demographics and peri/postoperative clinical and radiological features. Early and late radiological AsCF were defined as presentation before and after 3 months, respectively. We analyzed patients with AsCF for risk factors and survival duration by performing competing risk regression analyses where AsCF was the event of interest, with SF and death as competing events.

Results: We observed AsCF in 41/246 patients (16.7\%). The mean time to onset of AsCF after MSTS was 2 months (range, 1-9 months). Median survival of patients with AsCF was 20 and 41 months for early and late failures, respectively. Early AsCF accounted for $80.5 \%$ of cases, while late AsCF accounted for $19.5 \%$. The commonest radiologically detectable AsCF mechanism was angular deformity (increase in kyphus) in 29 patients. Increasing age $(p<0.02)$ and primary breast $(13 / 41,31.7 \%)(p<0.01)$ tumors were associated with higher AsCF rates. There was a non-significant trend towards AsCF in patients with a spinal instability neoplastic score $\geq 7$, instrumentation across junctional regions, and construct lengths of 6-9 levels. None of the patients with AsCF underwent revision surgery.

Conclusions: AsCF after MSTS is a distinct entity. Most patients with early AsCF did not require intervention. Patients who survived and maintained ambulation for longer periods had late failure. Increasing age and tumors with a better prognosis have a higher likelihood of developing AsCF. AsCF is not necessarily an indication for aggressive/urgent intervention.

Keywords: Spine; Neoplasm metastasis; Surgery; Artificial implants; Implant failure

Received Apr 13, 2020; Revised May 10, 2020; Accepted Jun 9, 2020

Corresponding author: Naresh Kumar

Department of Orthopaedic Surgery, University Orthopaedics, Hand \& Reconstructive Microsurgery Cluster, 1E Kent Ridge Road, NUHS Tower Block, Level 11, Singapore 119228

Tel: +65-67725611, Fax: +65-67780720, E-mail: dosksn@nus.edu.sg 


\section{Introduction}

The currently accepted indications for surgical intervention in patients with metastatic spine disease (MSD) are significant neurological deficits, spinal instability, and unremitting pain [1-3]. Decompression and stabilization, the primary surgical techniques for metastatic spinal tumor surgery (MSTS), are usually performed with no specific attempt towards fusion [4], which, in these situations, is deemed impractical due to concerns such as poor graft beds, risk of tumor seeding of the bone graft donor site, postoperative chemotherapy (CT)/radiotherapy (RT), poor patient condition/nutritional status, and expected shorter survival durations [5-7]. Despite this, the symptomatic implant/construct failure (SF) rates reported after MSTS range from $1.9 \%$ to $16 \%$ [7-14], significantly lower than SF rates for post-degenerative spine surgery $(4 \%-44 \%)[15,16]$.

Clinical presentations of SF such as neurological deficit, reduced ambulatory status, intractable pain, and any combination of the three can result in revision surgery. A subset of MSTS patients presenting with no symptoms despite radiological signs of construct failure (CF) $[9,10,17]$ was observed with a condition we term "asymptomatic CF” (AsCF) [7]. Amankulor et al. [9] observed a group of patients who showed CF but were asymptomatic; however, the authors did not elaborate except to mention that these patients behaved differently from similar patients with hardware failure in degenerative spine disease. The authors did not clearly explain the utility and importance of this phenomenon [7,9]. The exact incidence of AsCF is not unknown, given that existing literature on post-MSTS implant failure (IF) is mainly based on the incidence of clinically significant SF or hardware failure requiring revision surgery [7,9].

The descriptive nomenclature and terminology of IF/CF in MSTS have recently been established in the literature, but the clinical course of IF/CF is still poorly understood [7]. It is generally conjectured that AsCF represents one end of a continuum and, over time, develops into SF, the other end of the continuum. However, this perception has not been subjected to rigorous scientific consideration. AsCF could be a new entity that might not always develop into SF, thereby requiring no further intervention. Currently, the management of patients with AsCF remains uncertain and could potentially lead to unjustified alarm, unwarranted investigations, and unnecessary surgery.
The aim of this study was therefore to (1) study the incidence and onset of post-MSTS AsCF, (2) define the underlying mechanisms and associated factors leading to $\mathrm{AsCF}$, and (3) determine the clinical course of AsCF.

\section{Materials and Methods}

\section{Subjects}

We retrospectively collected data on patients who underwent MSTS at National University Health System, Singapore between 2005 and 2015. After obtaining ethical approval (DSRB ref no., 2014/00050), we accessed the patients' data through the hospital's computerized patient support system. Informed consent could not be obtained from patients due to the retrospective nature of the study design. The indications for MSTS were neurological deficits, spinal instability, intractable pain or any combination of the three. The exclusion criteria were (1) age $<18$ years, (2) prior spinal surgery for non-metastatic cause, (3) evidence of deep surgical site infections, and (4) expected survival of $<30$ days.

In our study, the surgical procedures were performed by one of five surgeons. The most common approach/ instrumentation was posterior for cervicothoracic, thoracic, thoracolumbar, lumbar, and lumbosacral procedures. Anterior reconstructions were performed through the posterior approach, except in two cases in which the reconstruction was performed through the anterior approach. The anterior or anterior with posterior approach was employed for the cervical procedures. Titanium pedicle screw systems and cages were employed for spinal stabilization and vertebral body replacement, respectively. Cement augmentation and cemented screws were not employed. Postoperative follow-ups occurred at approximately 2 weeks, 6 weeks, 3 months, 6 months, and every 6 months thereafter with clinical and radiological assessments. The endpoint of the assessments was either the last follow-up, point of revision surgery or death. The decision to perform revision surgery was based on clinical symptoms of SF that could be attributed to IF and/or loosening (e.g., pain, reduced mobility, and implant prominence) and on the expected survival at the time of SF detection.

We collected the patients' demographic details, primary tumor type, disease extent, pre/postoperative neurological status, ambulatory status, Eastern Cooperative Oncology Group (ECOG) score and indications for surgery (Tables 
Table 1. Demographic and clinical data of all patients

\begin{tabular}{|c|c|c|c|}
\hline Characteristic & Category & Total patients $(\%)^{\mathrm{a})}$ & Patients with asymptomatic failure $(\%)^{\mathrm{b})}$ \\
\hline Overall & & $246(100.0)$ & $41(16.7)$ \\
\hline Age (yr) & & $58.8 \pm 11.7$ & $62 \pm 10$ \\
\hline \multirow[t]{2}{*}{ Gender } & Male & $127(51.6)$ & $22(17.3)$ \\
\hline & Female & $119(48.4)$ & $19(15.9)$ \\
\hline \multirow[t]{5}{*}{ Primary tumor } & Lung & $68(27.6)$ & $8(11.7)$ \\
\hline & Breast & $41(16.7)$ & $13(31.7)$ \\
\hline & Prostate & $22(8.9)$ & $7(31.8)$ \\
\hline & Hematological & $30(12.2)$ & $7(23.3)$ \\
\hline & Others ${ }^{c)}$ & $85(34.6)$ & $6(7.0)$ \\
\hline \multirow[t]{3}{*}{ Type of lesion } & Sclerotic & $29(11.8)$ & $6(20.6)$ \\
\hline & Lytic & $163(66.2)$ & $25(15.3)$ \\
\hline & Mixed & $54(22.0)$ & $10(18.5)$ \\
\hline \multirow[t]{3}{*}{ SINS } & $<7$ (deemed stable) & $26(10.6)$ & $4(15.3)$ \\
\hline & 7-12 (indeterminate) & $178(72.4)$ & $31(17.4)$ \\
\hline & $>12$ (deemed unstable) & $42(17.0)$ & $6(14.2)$ \\
\hline \multirow[t]{2}{*}{ Preoperative ECOG scores } & $0-2$ & $46(18.7)$ & $6(13.0)$ \\
\hline & $3-4$ & $200(81.3)$ & $35(17.5)$ \\
\hline \multirow[t]{7}{*}{ Level of vertebra affected } & Cervical & $22(8.9)$ & $3(13.6)$ \\
\hline & Cervical-thoracic & $18(7.3)$ & $2(11.1)$ \\
\hline & Thoracic & $106(43.1)$ & $19(17.9)$ \\
\hline & Thoracolumbar & $32(13.0)$ & $6(18.7)$ \\
\hline & Lumbar & $52(21.1)$ & $10(19.2)$ \\
\hline & Lumbosacral & $6(2.4)$ & - \\
\hline & Multi-region involvement & $10(4.1)$ & $1(10.0)$ \\
\hline \multirow[t]{6}{*}{ Instrumented levels } & Occipito-cervical & $12(4.3)$ & $3(25.0)$ \\
\hline & Cervical-thoracic & $42(17.1)$ & $2(4.7)$ \\
\hline & Thoracic & $74(29.7)$ & $15(20.2)$ \\
\hline & Thoracolumbar & $83(33.7)$ & $16(19.2)$ \\
\hline & Lumbar & $15(6.1)$ & $1(6.6)$ \\
\hline & Lumbosacral/thoraco-lumbo-sacral & $20(8.1)$ & $4(20.0)$ \\
\hline \multirow[t]{2}{*}{ Skip instrumentation level } & $0-1$ & $130(56.0)$ & $20(15.0)$ \\
\hline & $\geq 2$ & $102(44.0)$ & $21(21.0)$ \\
\hline \multirow[t]{3}{*}{ Construct length } & $<6$ & $92(37.4)$ & $15(16.3)$ \\
\hline & $6-9$ & $123(49.3)$ & $23(18.6)$ \\
\hline & $>9$ & $31(12.6)$ & $3(9.6)$ \\
\hline \multirow[t]{2}{*}{ MIS or open approach } & MIS & $49(19.9)$ & $6(12.2)$ \\
\hline & Open & $194(78.9)$ & $35(18.0)$ \\
\hline
\end{tabular}

Values are presented as number (\%) or mean \pm standard deviation.

SINS, spinal instability neoplastic score; ECOG, Eastern Cooperation Oncology Group; MIS, minimally invasive surgery.

alIndicates percentage of cohort to total patients. ${ }^{b}$ Indicates percentage of failures within the cohort. ${ }^{c}$ Others include renal malignancies $(n=23)$, hepatocellular carcinoma/gastrointestinal tumors $(n=27)$, ovarian $(n=7)$, cervical $(n=9)$, thyroid $(n=7)$, nasopharyngeal $(n=10)$, and others $(n=2)$. 
Table 2. Primary tumor types and failure rates of each tumor type

\begin{tabular}{lccc} 
Primary tumor type & \multicolumn{1}{c}{ Total } & Asymptomatic failure (\% of failure within each cohort) & Asymptomatic failure (\% contributed by each type of tumor) \\
\hline Lung & $27.6(68 / 246)$ & $11.7(8 / 68)$ & $19.5(8 / 41)$ \\
\hline Breast & $16.7(41 / 246)$ & $31.7(13 / 40)$ & $31.7(13 / 41)$ \\
\hline Prostate & $8.9(22 / 246)$ & $31.8(7 / 22)$ & $17.1(7 / 41)$ \\
\hline Hematological & $12.2(30 / 246)$ & $23.3(7 / 30)$ & $17.1(7 / 41)$ \\
\hline Others $^{\text {a) }}$ & $34.6(85 / 246)$ & $11.7(6 / 85)$ & $14.6(6 / 41)$ \\
\hline
\end{tabular}

Values are presented as \% (number/total number).

${ }^{\text {al }}$ Others include renal malignancies $(n=23)$, hepatocellular carcinoma/gastrointestinal tumors $(n=27)$, ovarian $(n=7)$, cervical $(n=9)$, thyroid $(n=7)$, nasopharyngeal $(n=10)$, and others ( $n=2)$.

1-3). We studied the radiological characteristics of the metastatic spinal tumors in terms of the lytic, sclerotic or mixed pattern, and the vertebral levels involved. We determined the spinal instability neoplastic scores (SINS) using the available clinical and radiological data. The operative variables included the surgical approach (anterior/ posterior/combined), type of implant, and the spinal levels instrumented/skipped and/or decompressed. Pain was assessed pre- and postoperatively using the Visual Analog Scale. Postoperative radiographs were primarily used to assess implant/construct integrity and local disease progression. IFs/CFs were evaluated radiologically and documented as screw failure (ploughing, loosening, cut-out, pull-out, and breakage), cage failure (subsidence/tilting/ translation or breakage), rod breakage or changes in overall sagittal angulation $>5^{\circ}[7]$. In our series, most patients underwent conventional RT and/or CT approximately 2-3 weeks post-MSTS.

\section{Statistical analyses}

We conducted the statistical tests using Stata statistical software ver. 15.0 (Stata Corp., College Station, TX, USA). We employed Kaplan-Meier survival curves and Cox regression models to assess the mortality of the patients with AsCF, SF, and those without IF (Fig. 1). To determine the statistical differences between the various tumor groups, we employed log-rank tests (Fig. 2), initially applying a standard Cox proportional hazards model (Table 4). We graphically assessed the proportionality assumption of the Cox regression model using Schoenfeld residuals by means of the "estat phtest" STATA command after fitting a model with stcox [18]. Lastly, we employed the competing risk regression method to estimate the impact of the various tumor types on the probability of AsCF, where death and SF were treated as competing events. The results are expressed as subhazard ratios. We adjusted for various demographic factors, surgical factors, stability (SINS), and mobility (ECOG score). A $p$-value $<0.05$ was considered significant.

\section{Definitions}

IF is the term commonly used in the literature and is defined as radiologically detectable implant disassembly or breakage. We defined CF as radiologically detectable changes in construct appearance or integrity (implant or bone). In our article, we have used the term CF to represent both IF and $\mathrm{CF}$, because in most of the cases of MSD, the entire construct (consisting of the implant and implant bone interface) failed rather than just the implant alone. IF/CF could possibly develop through one or more of the various mechanisms previously described by Kumar et al. [7] in 2018. The failure mechanism of the various implant components (e.g., screw ploughing, screw loosening, and screw cut-through) have been described in the Supplemental Table 1, which has been adapted from the published article by Kumar et al. [7].

We defined SF as IF/CF in patients who exhibit pain, neurological deficit, and/or deterioration in their mobility from their peak postoperative mobility status [7]. We defined AsCF as radiologically detectable changes in the construct appearance or integrity (implant and/or bone), without pain, neurological deficit or deterioration in their mobility from their peak postoperative mobility status. We defined "early failure" as the onset of clearly detectable radiological signs of CF within 3 months of the index surgery, and we defined "late failure" as the onset of these signs after 3 months. 


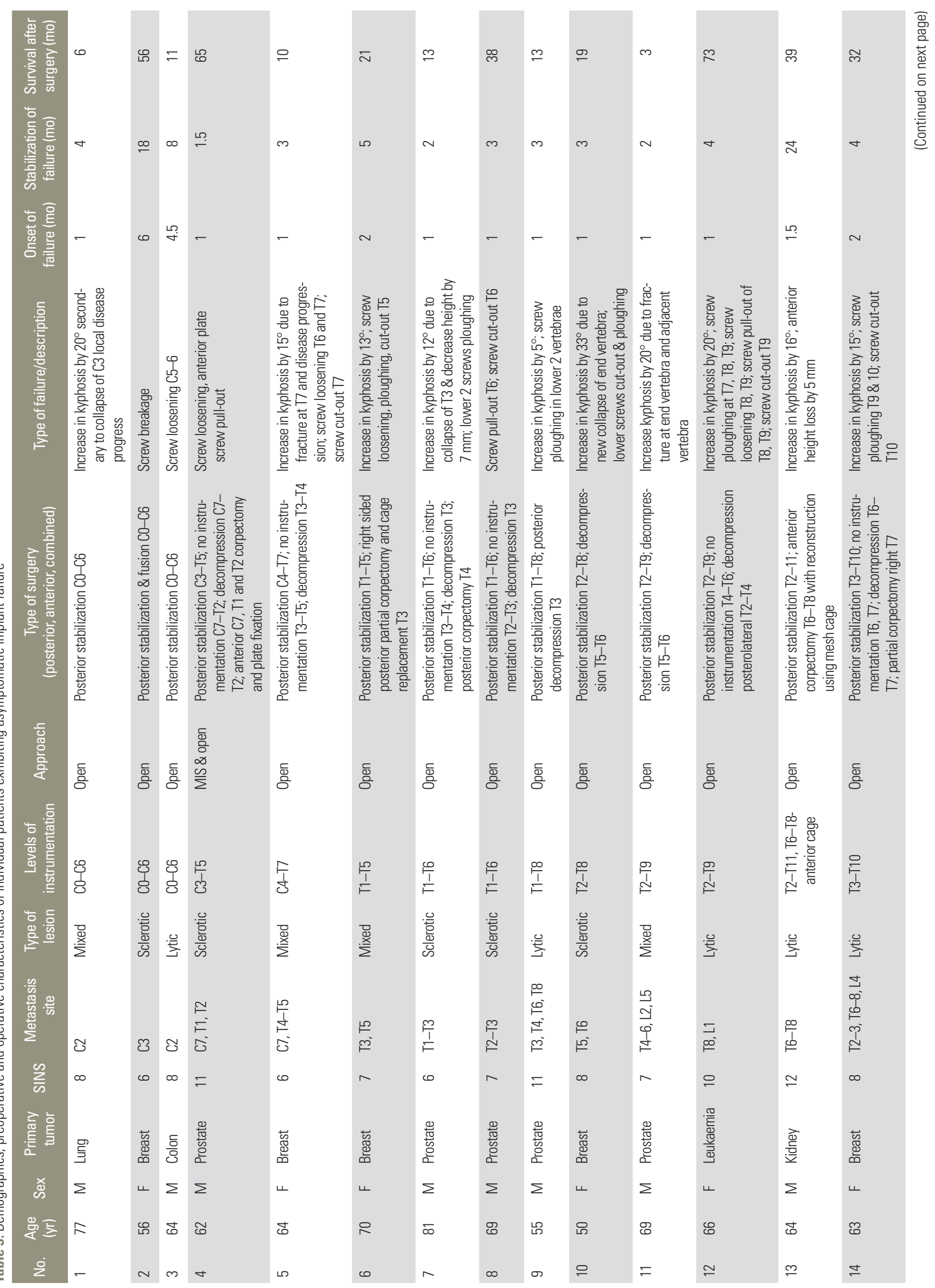




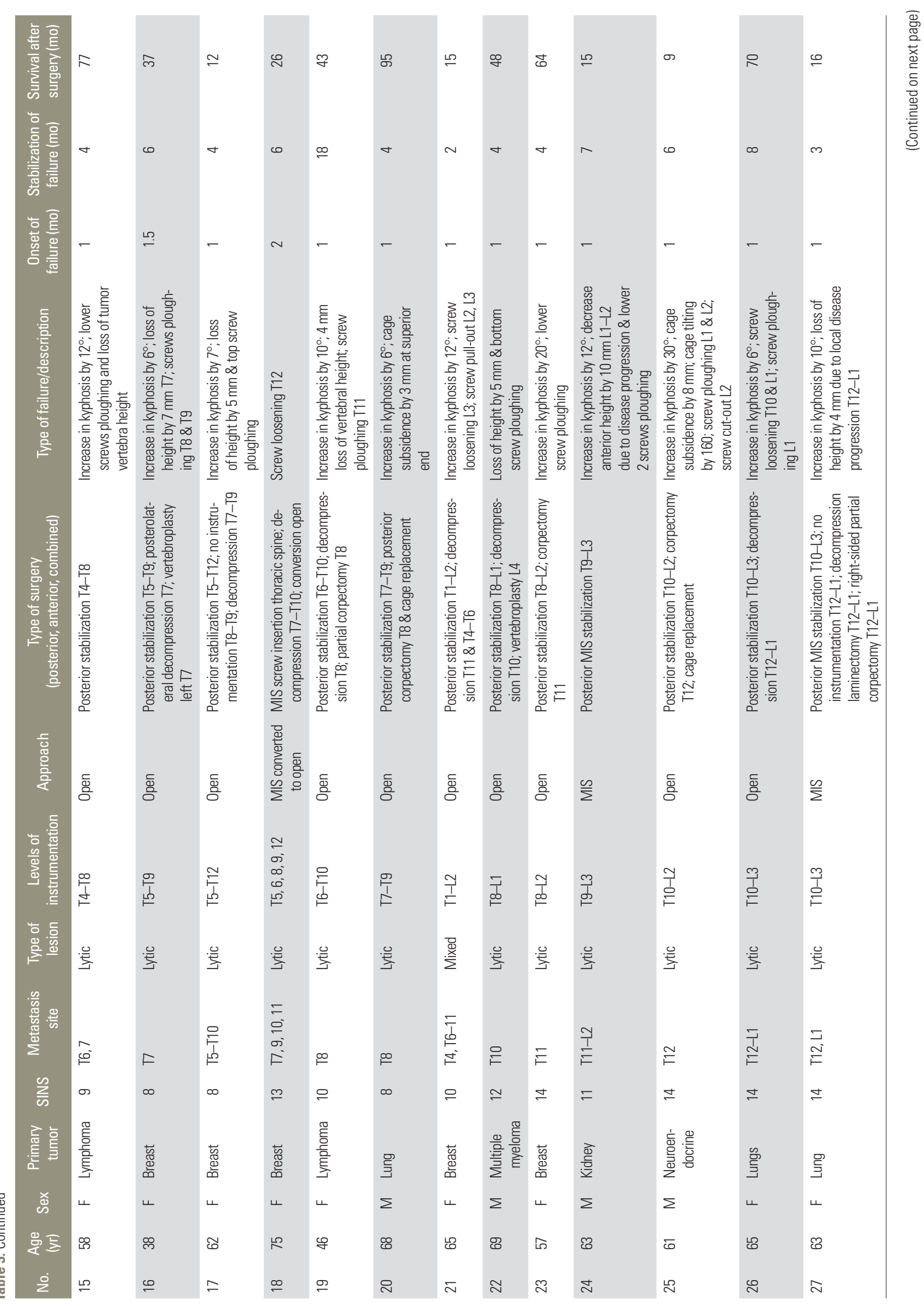




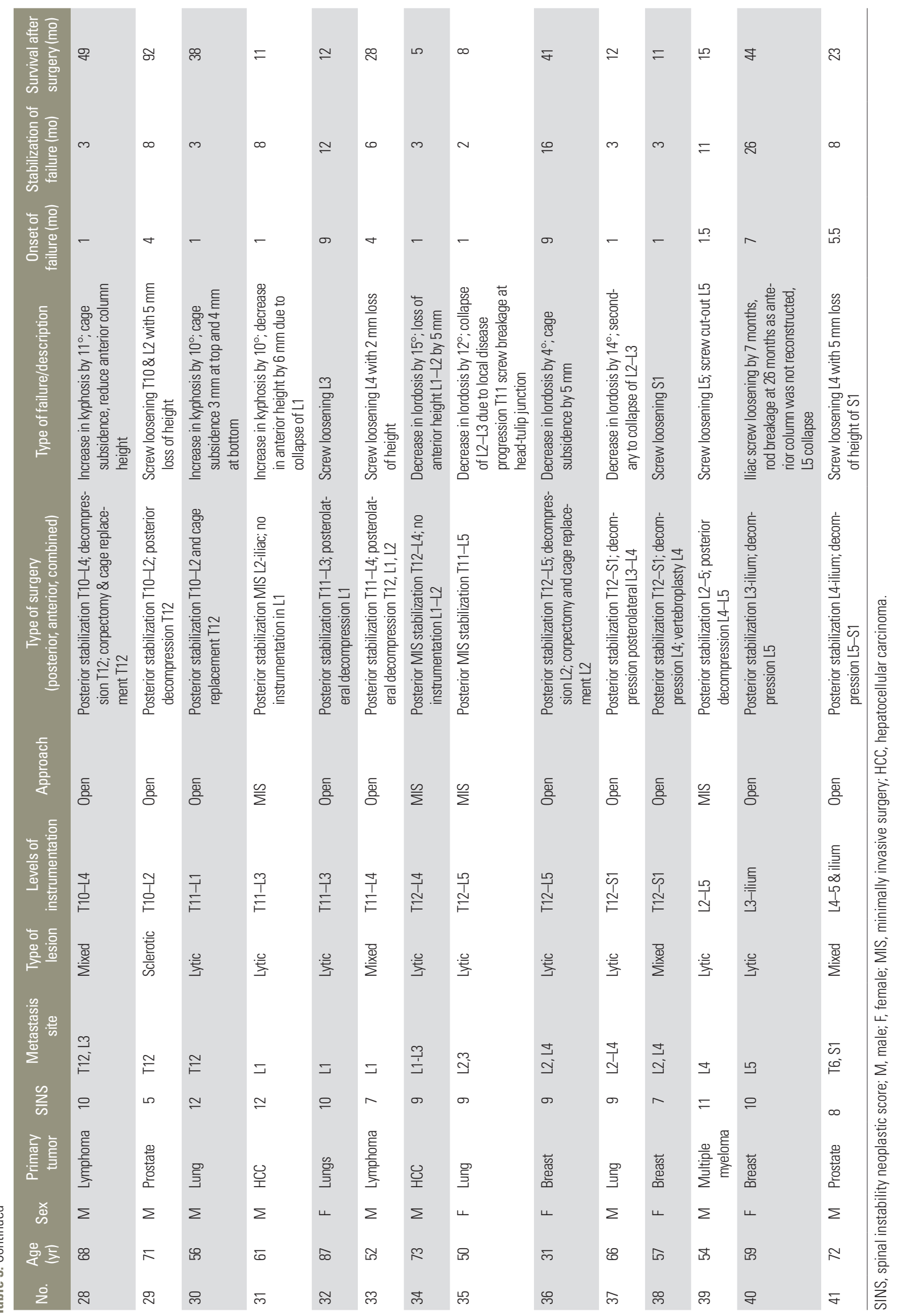


Table 4. Adjusted SHR estimate for asymptomatic failure and competing events (death or symptomatic failure) using multivariate competing risk regression analysis

\begin{tabular}{|c|c|c|}
\hline \multirow{2}{*}{ Variable } & \multicolumn{2}{|c|}{ Multivariate analysis } \\
\hline & Adjusted SHR (95\% CI) & $p$-value \\
\hline Age & $1.033(1.006-1.062)$ & 0.02 \\
\hline \multicolumn{3}{|l|}{ Sex } \\
\hline Male & 1.0 & \\
\hline Female & $1.87(0.719-4.901)$ & 0.19 \\
\hline \multicolumn{3}{|l|}{ Skipped region } \\
\hline 0 & 1.0 & \\
\hline 1 & 0.861 (0.311-2.388) & 0.77 \\
\hline 2 & $2.149(0.666-6.926)$ & 0.2 \\
\hline$\geq 3$ & $1.263(0.233-6.837)$ & 0.78 \\
\hline \multicolumn{3}{|l|}{ Metastatic region } \\
\hline Cervical & 1.0 & \\
\hline Cervicothoracic & $0.814(0.092-7.173)$ & 0.85 \\
\hline Thoracic & $0.928(0.188-4.577)$ & 0.92 \\
\hline Thoracolumbar & $1.263(0.186-8.578)$ & 0.81 \\
\hline Lumbar & $1.604(0.235-10.919)$ & 0.62 \\
\hline Lumbosacral & $0.419(0.038-4.576)$ & 0.47 \\
\hline \multicolumn{3}{|l|}{ Primary tumor } \\
\hline Breast & 1.0 & \\
\hline Lung & $0.142(0.044-0.458)$ & 0.001 \\
\hline Prostate & $0.113(0.020-0.621)$ & 0.01 \\
\hline Hematological & $0.230(0.064-0.813)$ & 0.02 \\
\hline Others ${ }^{\text {a) }}$ & $0.220(0.065-0.748)$ & 0.01 \\
\hline SINS & 0.992 (0.861-1.143) & 0.91 \\
\hline \multicolumn{3}{|l|}{ Lesion type } \\
\hline Sclerotic & 1.0 & \\
\hline Lytic & $0.706(0.228-2.185)$ & 0.54 \\
\hline Mixed & $0.822(0.322-2.0999)$ & 0.68 \\
\hline \multicolumn{3}{|l|}{ Instrumented region } \\
\hline Cervicothoracic & 1.0 & \\
\hline Thoracic & $1.860(0.522-6.620)$ & 0.33 \\
\hline Thoracolumbar & $2.697(0.801-9.080)$ & 0.10 \\
\hline Lumbar \& lumbosacral & $0.878(0.157-4.897)$ & 0.88 \\
\hline Construct length & 0.899 (0.696-1.162) & 0.41 \\
\hline \multicolumn{3}{|l|}{ ECOG score } \\
\hline 3-4 (non-ambulatory) & 1.0 & \\
\hline 0-2 (ambulatory) & 0.725 (0.290-1.809) & 0.49 \\
\hline
\end{tabular}

Bold type is considered statistically significant.

SHR, subhazard ratio; $\mathrm{Cl}$, confidence interval; SINS, spinal instability neoplastic score; ECOG, Eastern Cooperative Oncology Group.

${ }^{a}$ Others include renal malignancies $(n=23)$, hepatocellular carcinoma/gastrointestinal tumors $(n=27)$, ovarian $(n=7)$, cervical $(n=9)$, thyroid $(n=7)$, nasopharyngeal $(n=10)$, and others $(n=2)$.

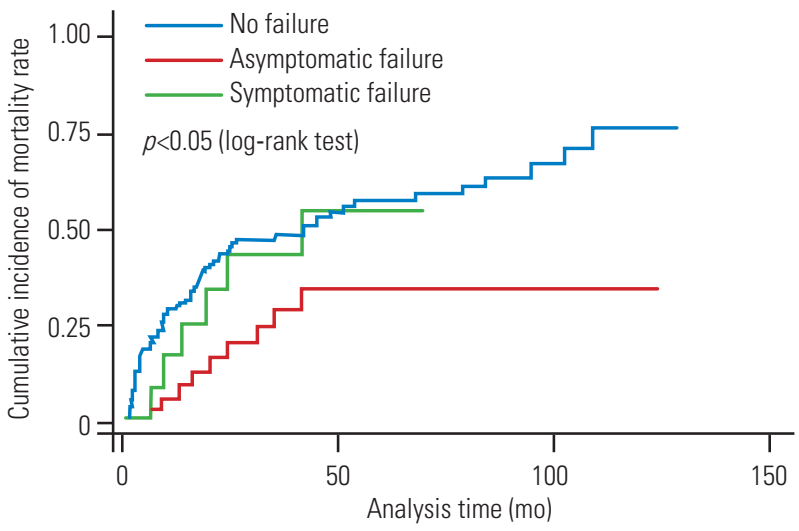

Fig. 1. Kaplan-Meier analysis of the survival between asymptomatic failure, symptomatic failure and no failures. Log-rank test $(p<0.05)$ indicates that the survival in asymptomatic failure group is more than symptomatic failure and death.

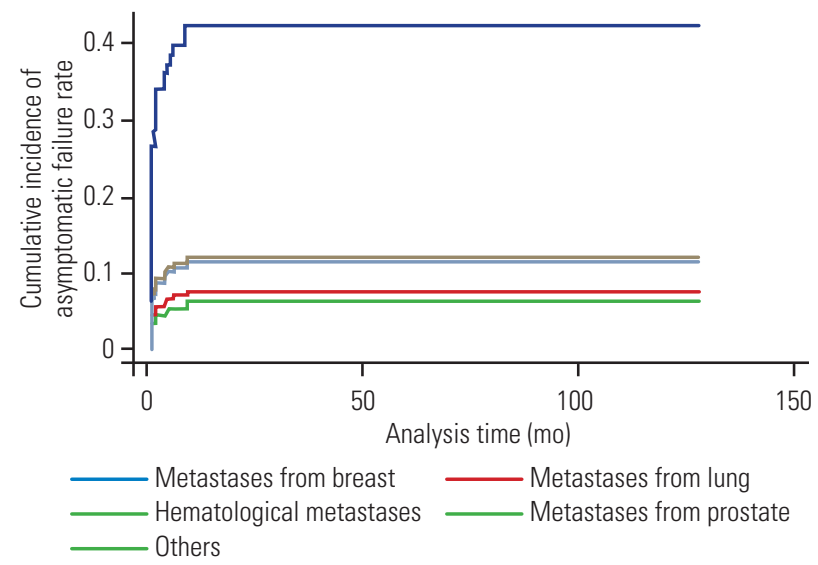

Fig. 2. Multivariate competing risk regression curve with asymptomatic failure as event of interest, with symptomatic failure and death as competing events. Primary tumor type (breast) was a significant risk factor for asymptomatic failure $(p<0.01)$.

\section{Results}

A total of 288 patients underwent MSTS between 2005 and 2015. We excluded 42 patients due to death within a period of 30 days after surgery or when their operation involved no instrumentation, leaving 246 patients for the final analysis. Table 1 summarizes the patients' demographics, preoperative, and operative characteristics. Of the 246 patients, we observed a total of 55 (23.4\%) CFs, of which there were 14 (25.5\%) SFs and 41 (74.5\%) AsCFs. All As$\mathrm{CFs}$ were CFs except for one who experienced hardware (rod) breakage and remained asymptomatic until he died. Table 2 describes the primary tumor types and their associated AsCF rates. Table 3 presents the individual clinical 
data for the patients who presented AsCF, with detailed descriptions of their failure mechanisms and preoperative characteristics. The rates for the various radiological failure mechanisms were $15 \%$ for screw ploughing, $15 \%$ for screw loosening, $3 \%$ for screw pull-out, $8 \%$ for screw cutout, $2 \%$ for screw breakage, $6 \%$ for cage subsidence, $1 \%$ for cage displacement-1, $0 \%$ for cage breakage, $1 \%$ for rod breakage, and, in terms of angular deformity, $25 \%$ for increase in kyphosis and $4 \%$ for decrease in lordosis. One or more mechanisms can exist in each AsCF case. The most common radiologically detectable failure mechanism in the AsCF group was angular deformity (increase in kyphosis) in 29 patients (71\%), followed by screw loosening and screw ploughing in 15 patients (37\%) each (Fig. 3).

The mean age at the time of surgery was 59 years (range, 21-87 years), with a sex distribution of 127 males (51.6\%) and 119 females (48.4\%). The common primary tumor types were lung (68 cases, $27.6 \%)$, breast $(41,16.7 \%)$, hematological $(30,12.9 \%)$, hepatocellular carcinoma/gastrointestinal $(27,10.9 \%)$, kidney $(23,9.3 \%)$, and prostate $(22$, $8.9 \%)$. The majority of lesions were lytic (66.3\%). Table 1 shows the instrumented regions, number of instrumentation levels, and the number of skipped vertebrae in the constructs. The main symptom at the first presentation prior to surgery was instability pain (95\%) followed by rest pain (49\%) and neurological deficit (47\%).

The mean time to the postoperative onset of AsCF was 2 months (range, 1-9 months). Early AsCF accounted for $80 \%(n=33)$ of the cases, while late AsCF accounted for $20 \%(n=10)$. By examining the early AsCFs, we found that they occurred due to (1) a reduction in height of the vertebra within the construct in patients who underwent only posterior instrumentation $(\mathrm{n}=22)$ and (2) cage subsidence/tilting in patients who underwent anterior column reconstruction ( $\mathrm{n}=11$ ) (Fig. 4).

The median survival for the entire cohort was 13.4 months, and the median survival for those with AsCF was 21.5 months (range, 3-123 months). The median survival for the patients with early and late AsCF was 20 months (range, 3-95 months) and 41 months (range, 11-92 months), respectively. The mean follow-up duration was 20 months, and $63 \%$ of the patients died during the follow-up period. The Kaplan-Meier survival curves showed that the survival time for the patients with AsCF was significantly longer than for patients with no failure or SF $(p<0.05)$ (Fig. 1$)$.

AsCFs were more common in the patients with breast
$(13 / 40,31.7 \%)$, prostate $(7 / 22,31.8 \%)$, and hematological $(7 / 30,23.3 \%)$ tumors. We observed a lower incidence among the patients with lung $(8 / 68,11.7 \%)$, gastrointestinal/hepatocellular carcinoma $(3 / 27,11.1 \%)$, and renal $(2 / 23,8.6 \%)$ tumors (Table 2$)$. Lytic metastatic lesions were the most common type $(25,61 \%)$, followed by mixed lesions (10, 24\%) and sclerotic metastases (6, 15\%). AsCF was common with SINS $\geq 7$ and was observed in one-sixth of the patients in each subcategory of the SINS classification (15.3\% [4/26] for stable [SINS 0-6], 17.4\% [31/178] for indeterminate instability [SINS 7-12] , and $14.2 \%$ [6/42] for the unstable groups [SINS 13-18]). The majority of AsCFs occurred in the instrumentation spanning the junctional regions $(25 / 41,61 \%)$. We observed that $25 \%(3 / 12)$ of the occipital-cervical fixations, $20 \%$ $(4 / 20)$ of the thoraco-lumbosacral/lumbosacral fixations, and $19.2 \%(16 / 83)$ of the thoracolumbar fixations developed AsCF. Most of the patients with AsCF had construct lengths of 6-9 levels (23/41,56\%). We observed AsCF in $12.2 \%(6 / 49)$ of the patients who underwent the minimally invasive surgical (MIS) approach, in contrast to the $18 \%$ (35/194) who underwent the open approach, although the difference was not statistically significant. We observed that most of the patients with AsCF were non-ambulatory prior to the surgery, with 35 of 41 patients (85\%) presenting a high ECOG score of (3-4). Based on the competing risk regression model multivariate analysis with death and SF as the competing events, we observed that primary tumors and increasing age $(p<0.02)$ were statistically significant risk factors for AsCF $(p<0.05)$ (Fig. 2, Table 4). We also found that breast tumors were the risk factor $(p<0.01)$ most strongly associated with AsCF (Fig. 2). None of the other factors considered in the multivariate analysis were statistically significant. None of the patients with AsCF underwent revision surgery during the study period.

\section{Discussion}

Most of the related studies in the literature have reported SF following MSTS, with reported SF rates of 1.9\%-16\% in the larger series of patients who underwent MSTS based on clinical presentation and the need for revision surgery $[8-14,19,20]$. Few studies have recognized patients who presented radiological CF while remaining clinically asymptomatic, and the exact incidence rate has not been reported $[9,10]$, which highlights the significant group of MSTS patients who are asymptomatic while exhibiting 

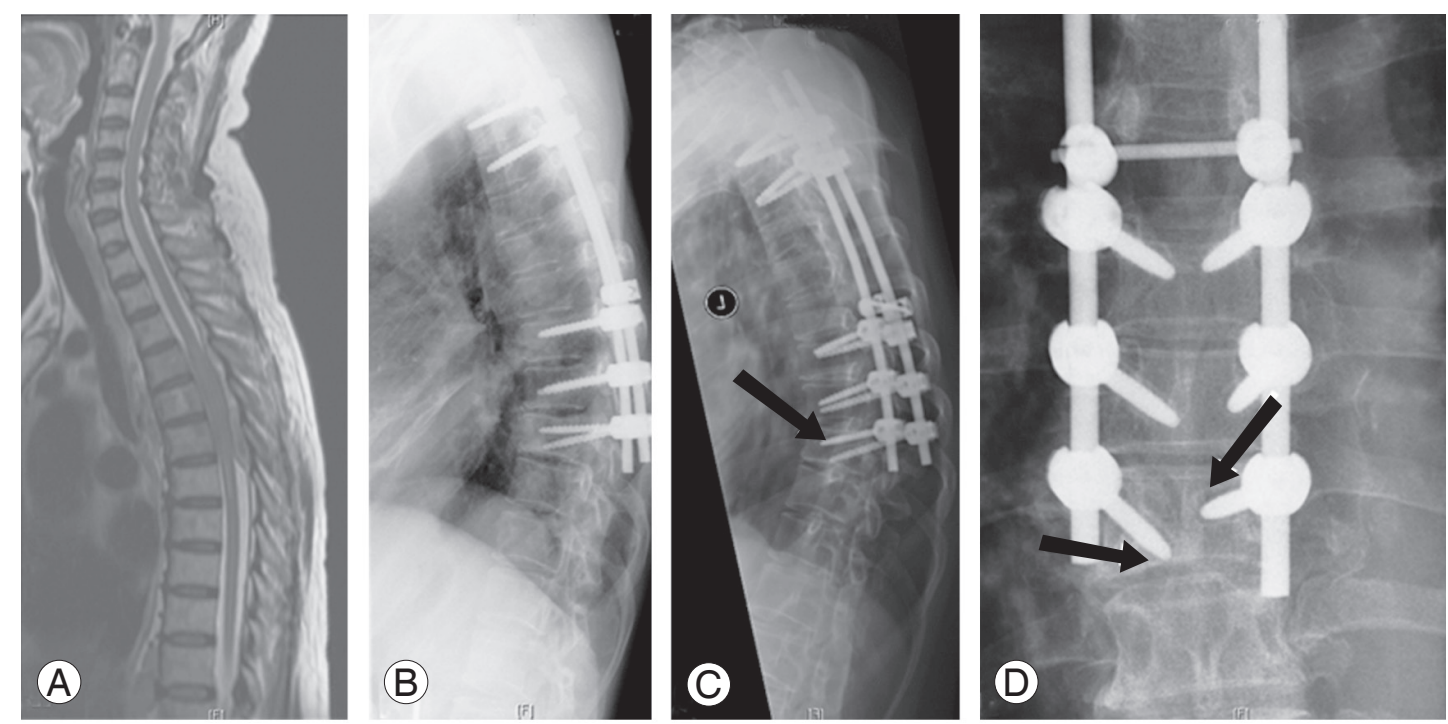

Fig. 3. (A) Spinal metastases to T4-T6 from chronic myeloid leukemia in 66-year-old male patient. (B) Posterior fixation from T2-T9 and decompression of T4-T6. (C, D) Late postoperative failure showing increase kyphosis by $20^{\circ}$; screw ploughing \& loosening in T8 and T9 presented 4 months after index surgery (arrows); total survival of 73 months.
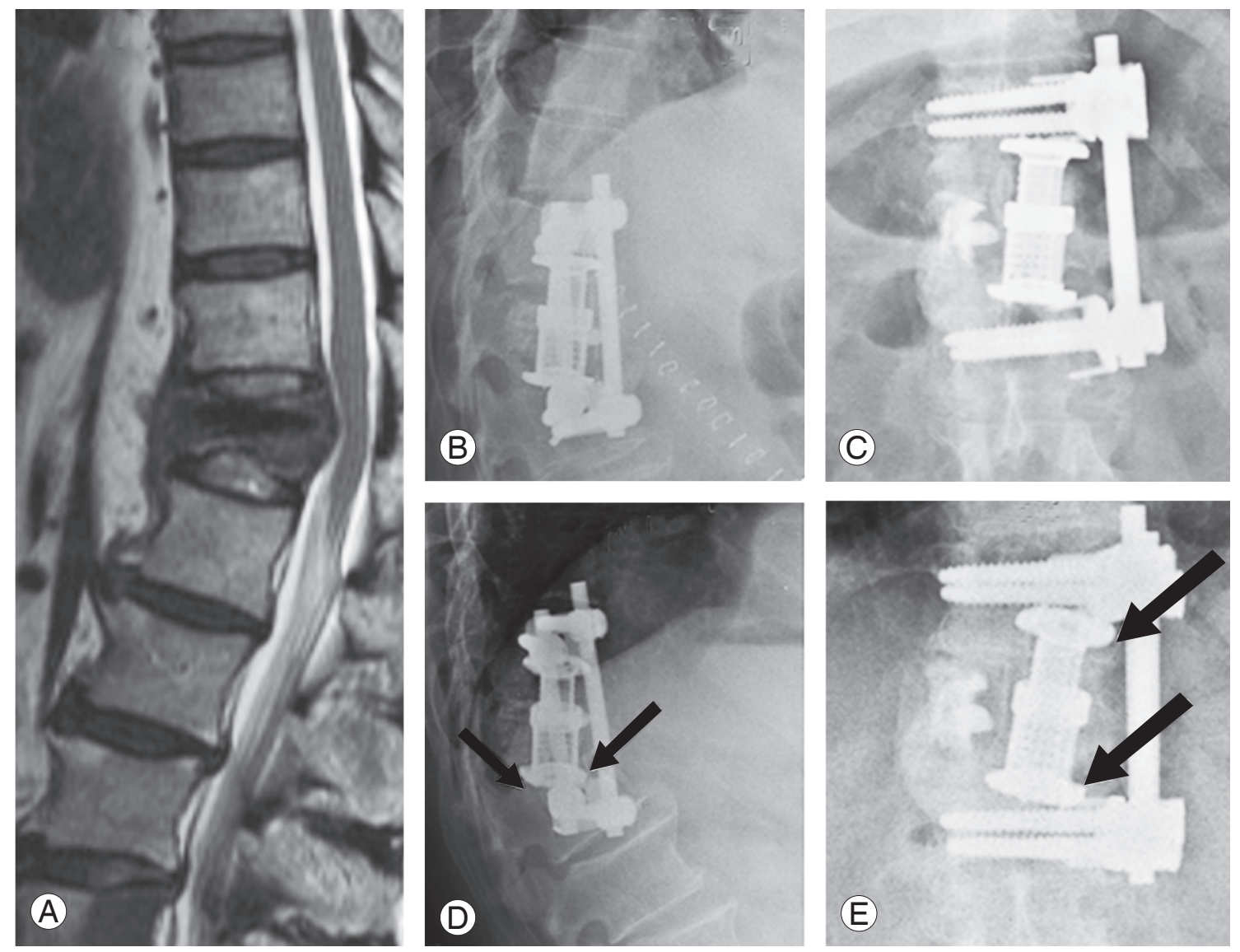

Fig. 4. (A) Spinal metastases to T12 from lungs in 56-year-old female patient. (B, C) Anterior decompression and reconstruction using cage and anterior fixation from T11-L1. (D, E) Early failure with increase in kyphosis by 10', cage sinkage by $7 \mathrm{~mm}$ presented within 1 month (arrows); total survival of 38 months. 
radiological signs of IF/CF.

Our study is the first to identify and report AsCF in a large series of MSTS patients. In our study, we observed AsCF in $16.7 \%$ of the patients following MSTS, a higher rate than the reported SF rates of $2.8 \%-8 \%$ in major studies [9-12]. Of the 41 patients in our series, we observed early AsCF and late AsCF in approximately $80 \%$ and $20 \%$, respectively. This large proportion of patients with early AsCF could be explained by an interesting observation in the literature: successful local disease control via RT causes re-calcification of affected vertebrae approximately 1-2 months after the start of RT [21,22]. This increase in strength of the diseased bone after treatment could improve the construct strength and pause the process of further collapse of the diseased vertebra, resulting in no further worsening of the radiological signs of CF. Three months post-MSTS would approximately coincide with the re-calcification of affected vertebrae, increasing their load-sharing capacity to provide support to the anterior column. Before the re-calcification of affected vertebrae, their load-sharing capacity would be suboptimal, thus the rationale for choosing 3 months as the distinction between early and late failures. The introduction of RT/CT and treatment of osteoporosis improves the bone mineral density and quality of the vertebrae, thereby reducing the possibility of early AsCF progressing to SF $[5,22,23]$. RTrelated re-calcification, as mentioned above $[21,22]$, could also be a plausible explanation for the low number of patients seen with AsCF in the MIS group compared with the open group (Table 1). The MIS patients conventionally underwent local RT 1-2 weeks earlier than the open group, thus resulting in earlier re-calcification of the diseased vertebra.

We observed that kyphosis occurred in the vertebrae when spanned by posterior instrumentation without anterior reconstruction, a condition that might have been caused by axial compressive forces. It is possible that the involved vertebrae had low initial bone mass coupled with microscopic tumor metastases. Anterior reconstruction using a cage/graft demonstrated similar patterns of collapse, possibly as a result of mild to moderate cage subsidence in the adjacent osteoporotic vertebral bodies. Cage subsidence or tilting persists until contact is made with resilient cancellous bone or screws. However, there is consolidation of the anterior column following both postoperative RT/CT and treatment of osteoporosis, resulting in stabilization of the kyphosis in an acceptable position.
Patients therefore remain asymptomatic despite radiological signs of failure.

In our retrospective study, we observed rates of $16.7 \%$ for $\mathrm{AsCF}$ and $5.7 \%$ for SF. Considering that most of the patients began as AsCF, it can be surmised that only $25 \%$ of the cases of AsCF progressed to SF, demonstrating that a continuation to $\mathrm{SF}$ is not always the case for AsCF. There is the possibility that an initial AsCF can consolidate and stabilize in an acceptable position after effective adjuvant therapy, arresting the progression of AsCF to SF. Attempts to fix AsCF might therefore lead to unnecessary operations in $75 \%$ of patients, given that potentially only $25 \%$ progress to SF. In patients with AsCF after MSTS, there is the possibility that an initial AsCF might consolidate and stabilize in the new acceptable position, after the effective completion of adjuvant therapy, thereby arresting the progression of AsCF to SF. In our study, we typically observed late AsCF in (1) the patients with primary tumors that had good prognoses (e.g., breast, prostate) with prolonged survival in the absence of fusion $(\mathrm{n}=6)$ and (2) the patients who initially responded to CT/RT for local disease control after the index surgery but later relapsed, leading to the loss of anterior column support $(\mathrm{n}=2)$. Only age $(p<0.02)$ and tumor type $(p<0.05)$ were statistically significant risk factors among the many factors considered (Table 4 ). The median survival for our entire cohort was 13.4 months (range, 1-129 months), while the median survival for the patients with late AsCF was 41 months. The Kaplan-Meir analysis of the survival between $\mathrm{AsCF}, \mathrm{SF}$ and no failure indicated that the survival for the AsCF group was longer than for SF and no failure $(p<0.05)$ (Fig. 1).

Studies have shown that survival is strongly influenced by factors such as primary tumor type and the patients' general condition $[24,25]$. Metastatic tumors with generally good prognoses (e.g., breast and prostate primary tumors) together comprised nearly $63 \%$ of the asymptomatic cohort (Table 2) and were therefore considered a significant risk factor for AsCF, with primary breast tumors the most significant risk factor, as shown by the multivariate competing risk regression curve $(p<0.01)$ (Fig. 2). These results imply that, for these patients, spine surgeons tend to more frequently discover asymptomatic IFs, which could be attributed to the fact that implants in these patients are subjected to cyclical loading in the absence of fusion, developing CF in the form of progressive kyphosis and screw cut-out/ploughing during the early postoperative months, which later stabilize when these 
tumors respond to RT/CT [5,21-23].

In our study, AsCF was most frequently observed in the junctional regions (61\%), with one-quarter in the occipital-cervical fixations, one-fifth in thoraco-lumbosacral/ lumbosacral fixations, and one-fifth in thoracolumbar fixations. This was probably due to the presence of higher mechanical stress, in the absence of fusion, in the junctional regions of the ambulatory patients. There was also a trend towards AsCF in the patients with SINS $>7$ and construct lengths of 6-9 levels; however, the multivariate regression analysis showed that the associations were not significant.

This concept was further validated by our observation that most of the patients with AsCF had a poor preoperative ambulatory status, with ECOG scores of 3-4 for $85 \%$ of the patients, implying that most of these patients were not adequately mobile during their early postoperative period. The reduced cyclical loading of implants due to reduced mobility resulted in certain radiological changes; however, the patients remained asymptomatic. This reduced ambulation provided an opportunity for re-ossification of the tumor-involved vertebra after the introduction of RT/CT. This re-ossification is a known phenomenon that occurs after the successful introduction of RT and/ or CT during the postoperative period following MSTS, as described by Greenburg et al. [21] who observed that it takes approximately 1-2 months for the affected vertebrae to re-calcify after starting $\mathrm{CT}$ and/or RT in patients with MSD [21].

Higher preoperative ECOG scores and lower early postoperative mobility might reduce the risk of early AsCF, a notion supported by Kondo et al. [26] who evaluated 96 non-ambulatory patients before MSTS and discovered that they experience no IF within a median follow-up of 7 months. However, if the patients' general condition is good at the time of the index surgery and continues to remain so during the survival period, the patients might present as late AsCF. The possible reasons for the lack of symptoms despite the discernible radiological signs of failure could be that these patients (1) have a higher threshold for reporting pain; (2) were already undergoing pain management for their primary tumors, thereby masking the pain due to CF; (3) might not have been sufficiently mobile to load the implants and therefore unable to provoke instability pain; and (4) have constructs that would have stabilized after the initial radiological failure due to re-ossification of the tumor-involved vertebrae, as alluded to earlier.

AsCF raises alarms among spine surgeons and results in more frequent follow-ups for these patients. However, most surgeons prefer not to address AsCF surgically, unless the patients become symptomatic or radiologically unstable; however, this observation is mainly anecdotal rather than evidence-based. Our study supports the initial non-interventional approach towards AsCF and recommends close follow-up of patients with AsCF. In the event of progression to SF, surgical intervention may be offered.

Surgical techniques and planning have evolved over the 10 years of our retrospective study, resulting in variability in the construct selection, surgical planning, and techniques. Further variations were introduced via the individual variation in the decision process of the five consultants performing the operations. Subsequent studies might show a lower incidence of IF than that reported in our study due to further refinement in the surgical techniques and implant design.

\section{Conclusions}

The new concept of AsCF after MSTS is a distinct entity that should be recognized. In most cases, AsCF can be detected early; however, few of those cases progress to SF. We observed early failures in $80 \%$ of the patients who presented with AsCF, which were noted in the patients with reduced ambulation in the postoperative period before the successful effect of CT/RT was noted in achieving local disease control. We observed late failures in patients with tumors with better prognoses (e.g., breast), advanced age and mobile patients. The observation of radiological failure during the postoperative period (early or late) should be followed up with caution, and aggressive surgical intervention should be resisted. We recommend more frequent follow-ups for these patients, with further investigation and careful consideration for surgery only in cases of progressive symptoms.

\section{Conflict of Interest}

No potential conflict of interest relevant to this article was reported.

\section{Acknowledgments}

We thank Dr. Aye Sandar Zaw for the preparation of "Table 
3" and statistical analysis. We thank Ms. Sirisha Madhu \& Mr. Sridharan Alathur Ramakrishnan for editing the final document.

\section{Supplementary Materials}

Supplementary materials can be available from https:// doi.org/10.31616/asj.2020.0167. Supplemental Table 1. Mechanisms of failure.

\section{References}

1. Bilsky MH, Laufer I, Burch S. Shifting paradigms in the treatment of metastatic spine disease. Spine (Phila Pa 1976) 2009;34(22 Suppl):S101-7.

2. Klimo P Jr, Schmidt MH. Surgical management of spinal metastases. Oncologist 2004;9:188-96.

3. Kumar N, Malhotra R, Zaw AS, et al. Evolution in treatment strategy for metastatic spine disease: presently evolving modalities. Eur J Surg Oncol 2017;43:1784-801.

4. Park SJ, Lee KH, Lee CS, et al. Instrumented surgical treatment for metastatic spinal tumors: is fusion necessary? J Neurosurg Spine 2019;22:1-9.

5. Georgiou KR, Scherer MA, Fan CM, et al. Methotrexate chemotherapy reduces osteogenesis but increases adipogenic potential in the bone marrow. J Cell Physiol 2012;227:909-18.

6. Sugimoto M, Takahashi S, Toguchida J, Kotoura Y, Shibamoto Y, Yamamuro T. Changes in bone after high-dose irradiation: biomechanics and histomorphology. J Bone Joint Surg Br 1991;73:492-7.

7. Kumar N, Patel R, Wadhwa AC, et al. Basic concepts in metal work failure after metastatic spine tumour surgery. Eur Spine J 2018;27:806-14.

8. Akeyson EW, McCutcheon IE. Single-stage posterior vertebrectomy and replacement combined with posterior instrumentation for spinal metastasis. J Neurosurg 1996;85:211-20.

9. Amankulor NM, Xu R, Iorgulescu JB, et al. The incidence and patterns of hardware failure after separation surgery in patients with spinal metastatic tumors. Spine J 2014;14:1850-9.

10. Bellato RT, Teixeira WG, Torelli AG, Cristante AF, de Barros TE, de Camargo OP. Late failure of posterior fixation without bone fusion for vertebral metastases. Acta Ortop Bras 2015;23:303-6.
11. Mesfin A, Sciubba DM, Dea N, et al. Changing the adverse event profile in metastatic spine surgery: an evidence-based approach to target wound complications and instrumentation failure. Spine (Phila Pa 1976) 2016;41 Suppl 20:S262-70.

12. Pedreira R, Abu-Bonsrah N, Karim Ahmed A, et al. Hardware failure in patients with metastatic cancer to the spine. J Clin Neurosci 2017;45:166-71.

13. Quraishi NA, Rajabian A, Spencer A, et al. Reoperation rates in the surgical treatment of spinal metastases. Spine J 2015;15(3 Suppl):S37-43.

14. Vrionis FD, Small J. Surgical management of metastatic spinal neoplasms. Neurosurg Focus 2003;15:E12.

15. Kim KT, Lee SH, Lee YH, Bae SC, Suk KS. Clinical outcomes of 3 fusion methods through the posterior approach in the lumbar spine. Spine (Phila Pa 1976) 2006;31:1351-7.

16. Mok JM, Cloyd JM, Bradford DS, et al. Reoperation after primary fusion for adult spinal deformity: rate, reason, and timing. Spine (Phila Pa 1976) 2009;34:8329.

17. Wise JJ, Fischgrund JS, Herkowitz HN, Montgomery D, Kurz LT. Complication, survival rates, and risk factors of surgery for metastatic disease of the spine. Spine (Phila Pa 1976) 1999;24:1943-51.

18. Fisher LD, Lin DY. Time-dependent covariates in the Cox proportional-hazards regression model. Annu Rev Public Health 1999;20:145-57.

19. Placantonakis DG, Laufer I, Wang JC, Beria JS, Boland P, Bilsky M. Posterior stabilization strategies following resection of cervicothoracic junction tumors: review of 90 consecutive cases. J Neurosurg Spine 2008;9:111-9.

20. Wang JC, Boland P, Mitra N, et al. Single-stage posterolateral transpedicular approach for resection of epidural metastatic spine tumors involving the vertebral body with circumferential reconstruction: results in 140 patients: invited submission from the Joint Section Meeting on Disorders of the Spine and Peripheral Nerves, March 2004. J Neurosurg Spine 2004;1:287-98.

21. Greenburg EJ, Chu FC, Dwyer AJ, Ziminski EM, Dimich AB, Laughlin JS. Effects of radiation therapy on bone lesions as measured by $47 \mathrm{Ca}$ and $85 \mathrm{Sr}$ local kinetics. J Nucl Med 1972;13:747-51.

22. Libshitz HI, Hortobagyi GN. Radiographic evalua- 
tion of therapeutic response in bony metastases of breast cancer. Skeletal Radiol 1981;7:159-65.

23. Cunha SS, Sarmento VA, Ramalho LM, et al. Effects of radiotherapy on bone tissues. Radiol Bras 2007;40:189-92.

24. Tokuhashi Y, Matsuzaki H, Oda H, Oshima M, Ryu J. A revised scoring system for preoperative evaluation of metastatic spine tumor prognosis. Spine (Phila Pa 1976) 2005;30:2186-91.
25. Zaw AS, Kantharajanna SB, Maharajan K, Tan B, Vellayappan B, Kumar N. Perioperative blood transfusion: does it influence survival and cancer progression in metastatic spine tumor surgery? Transfusion 2017;57:440-50.

26. Kondo T, Hozumi T, Goto T, Seichi A, Nakamura K. Intraoperative radiotherapy combined with posterior decompression and stabilization for non-ambulant paralytic patients due to spinal metastasis. Spine (Phila Pa 1976) 2008;33:1898-904. 